\title{
A Chromatic Dispersion Estimation Method for Arbitrary Modulation Formats
}

\author{
John Zweck ${ }^{1}$ and Curtis R. Menyuk ${ }^{2}$ \\ ${ }^{1}$ Department of Mathematics and Statistics \\ ${ }^{2}$ Department of Computer Science and Electrical Engineering \\ University of Maryland Baltimore County, Baltimore, Maryland 21250,USA \\ zweck@umbc.edu
}

\begin{abstract}
Simulations show that a modulation-format-independent method for estimating chromatic dispersion from the phase of a coherently-received signal at four frequencies can estimate $3000 \mathrm{ps} / \mathrm{nm}$ of dispersion to within $2 \%$ at an OSNR of $10 \mathrm{~dB}$.
\end{abstract}

(C) 2010 Optical Society of America

OCIS codes: $060.1660,060.2360,060.1155$.

\section{Introduction}

Recent advances in coherent receivers now allow for optical noise and chromatic dispersion (CD) to be monitored and mitigated using digital signal processing techniques [1,2]. Typically, compensation of CD is performed using electrical equalization filters that are continuously adapted using optimization algorithms such as minimum mean square estimation (MMSE) [1]. All such optimization methods use a priori knowledge of the optical data modulation format to define the cost function to be minimized. For example, with the constant modulus algorithm (CMA) [3], the data symbols are assumed to lie on a circle in the constellation diagram. Consequently, such methods often need to be individually designed for each format (see, for example, [4]), which is challenging — particularly in light of the recent proliferation of novel optical modulation formats [5].

In this paper, we introduce a method to estimate $\mathrm{CD}$ for arbitrary modulation formats impaired by arbitrary amounts of $\mathrm{CD}$ and noise. The method is based on an analytical formula for the CD in terms of the phase of the optical signal at four frequencies, and does not use optimization. We describe a preliminary version of the method and present initial simulation results demonstrating that the method is effective for a wide variety of formats even when the OSNR approaches the FEC limit. The method could be used to monitor performance in reconfigurable heterogeneous optical networks and in the design of general purpose coherent receiver systems.

\section{Theory}

If the signal is impaired only by $\mathrm{CD}$, then the Fourier transform, $\widetilde{u}$, of the coherently-received signal, $u$, is given by

$$
\widetilde{u}(\omega)=\widetilde{v}(\omega)\left(\sum_{n=0}^{N-1} b_{n} \exp (i \omega n T)\right) \exp \left(i \beta \omega^{2} / 2\right) \exp \left(i \theta_{0}+i \omega t_{0}\right),
$$

where $\omega$ is angular frequency, $v=v(t)$ is the pulse shape at the transmitter, $b_{n}$ is the $n$-th data symbol, $T$ is the symbol period, $\beta$ is the total dispersion, and $t_{0}$ and $\theta_{0}$ are unknown time and phase offsets. To determine the chromatic dispersion, $\beta$, we choose two frequencies, $\omega_{j}=2 \pi f_{j}$ for $j=1,2$, and define

$$
\Theta\left(f_{1}, f_{2}\right)=\arg \left[\widetilde{u}\left(f_{1}\right)\right]+\arg \left[\widetilde{u}\left(-f_{1}\right)\right]-\arg \left[\widetilde{u}\left(f_{2}\right)\right]-\arg \left[\widetilde{u}\left(-f_{2}\right)\right] .
$$

We assume that $\arg [\widetilde{v}(f)]$ is an even function of $f$, which, for example, holds if $v$ is real and even. Then, we find that

$$
\Theta\left(f_{1}, f_{2}\right)=4 \pi^{2} \beta\left(f_{1}^{2}-f_{2}^{2}\right)+\varphi_{b}\left(f_{1}\right)+\varphi_{b}\left(-f_{1}\right)-\varphi_{b}\left(f_{2}\right)-\varphi_{b}\left(-f_{2}\right)+2 k \pi, \quad k= \pm 1, \pm 2, \ldots,
$$

where

$$
\varphi_{b}(f)=\arg \left[\sum_{n=0}^{N-1} b_{n} \exp (2 \pi i f T n)\right]
$$


If the data symbols, $b_{n}$, are real, then $\varphi_{b}$ is an odd function and so $\Theta\left(f_{1}, f_{2}\right)=4 \pi^{2} \beta\left(f_{1}^{2}-f_{2}^{2}\right)+2 k \pi$. On the other hand, for complex-valued symbols, $b_{n}$, we may choose $f_{1}=(1 / 2 T)+f$ and $f_{2}=(1 / 2 T)-f$, for some frequency, $f$. We then find that $\varphi_{b}\left(f_{2}\right)=\varphi_{b}\left(-f_{1}\right)$ and $\varphi_{b}\left(f_{1}\right)=\varphi_{b}\left(-f_{2}\right)$, and so $\Theta(f)=\left(8 \pi^{2} f / T\right) \beta+2 k \pi$. For the results in this paper, we suppose that the absolute value of the dispersion does not exceed a maximum value given by

$$
|\beta|<\beta_{\max }=\frac{1}{4 \pi\left(f_{1}^{2}-f_{2}^{2}\right)}=\frac{T}{8 \pi f} .
$$

Then the dispersion is unambiguously given by

$$
\beta=\frac{\Theta\left(f_{1}, f_{2}\right)}{4 \pi^{2}\left(f_{1}^{2}-f_{2}^{2}\right)}=\frac{T \Theta(f)}{8 \pi^{2} f}, \quad \text { where }-\pi \leq \Theta<\pi .
$$

To mitigate for errors due to noise, we average the angle $\Theta$ in Eqn. (2) over many noise (and data) realizations. For the preliminary results presented here, we arranged for each realization of the signal to be periodic in time. The restrictions on the periodicity of the signal and the maximum allowed value of the disperison will be removed in a future paper.

\section{Simulation Results}

We tested the method on six formats generated using a Mach-Zehnder modulator (NRZ, RZ33 (33\% duty cycle), RZ50, CSRZ, BPSKMZ, and QPSKMZ) and on two formats generated using a phase modulator (BPSKPM and QPSKPM). We generated signals at a baud rate of $10 \mathrm{~Gb} / \mathrm{s}$, with OSNR values of of 5, 10, and $15 \mathrm{~dB}$, and with dispersions of $500 \mathrm{ps} / \mathrm{nm}$ and $3000 \mathrm{ps} / \mathrm{nm}$. For each format, OSNR, and dispersion value we generated 2048 periodic signals each with 64 symbols. For each signal, we used different realizations of the data symbols and noise. We used $f_{1}=6 \mathrm{GHz}$ and $f_{2}=4 \mathrm{GHz}$. The maximum dispersion was $\beta_{\max }=3120 \mathrm{ps} / \mathrm{nm}$. On the left in Table 1 , we show the errors in the estimated dispersion (in ps/nm) when the dispersion was $500 \mathrm{ps} / \mathrm{nm}$, and on the right when the dispersion was $3000 \mathrm{ps} / \mathrm{nm}$. The results suggest that the relative error in the overall estimate could be reduced to less than $2 \%$ for signals with $3000 \mathrm{ps} / \mathrm{nm}$ dispersion and an OSNR of at least $10 \mathrm{~dB}$ if we were to first compensate for the dispersion using the estimated dispersion values and then estimate the dispersion of these compensated signals.

Table 1. Error in the estimated dispersion (in $\mathrm{ps} / \mathrm{nm}$ ) displayed as a function of the modulation format (rows) and OSNR (columns). The total dispersion was $500 \mathrm{ps} / \mathrm{nm}$ (left) and $3000 \mathrm{ps} / \mathrm{nm}$ (right).

\begin{tabular}{|c||c|c|c|}
\hline Format & $5 \mathrm{~dB}$ & $10 \mathrm{~dB}$ & $15 \mathrm{~dB}$ \\
\hline \hline NRZ & 162 & 59 & 1 \\
\hline RZ33 & 14 & 17 & 27 \\
\hline RZ50 & 55 & 37 & 27 \\
\hline CSRZ & 6 & 20 & 42 \\
\hline BPSKMZ & 7 & 36 & 44 \\
\hline QPSKMZ & 30 & 25 & 41 \\
\hline BPSKPM & 107 & 29 & 3 \\
\hline QPSKPM & 109 & 20 & 18 \\
\hline
\end{tabular}

\begin{tabular}{|c||c|c|c|}
\hline Format & $5 \mathrm{~dB}$ & $10 \mathrm{~dB}$ & $15 \mathrm{~dB}$ \\
\hline \hline NRZ & 148 & 164 & 189 \\
\hline RZ33 & 183 & 186 & 175 \\
\hline RZ50 & 236 & 168 & 195 \\
\hline CSRZ & 226 & 212 & 187 \\
\hline BPSKMZ & 155 & 191 & 186 \\
\hline QPSKMZ & 191 & 205 & 179 \\
\hline BPSKPM & 204 & 154 & 198 \\
\hline QPSKPM & 75 & 160 & 191 \\
\hline
\end{tabular}

\section{References}

1. F. N. Hauske, M. Kushnerov, B. Spinnler, and B. Lank1, "Optical performance monitoring in digital coherent receivers," J. Lightw. Technol. 27, 3623-3631 (2009).

2. J. C. Geyer, C. R. S. Fludger, T. Duthel, C. Schulien, and B. Schmauss, "Performance monitoring using coherent receivers," in Proc. OFC'09, (San Diego, 2009), paper OThH5.

3. D. N. Goddard, "Self-recovering equalization and carrier tracking in two-dimensional data communication systems," IEEE Trans. on Commun. COM-28, 1867-1875 (1980).

4. X. Zhou, J. Yu, and P. Magill, "Cascaded two-modulus algorithm for blind polarization demultiplexing of 114 Gb/s PDM-8-QAM optical signals," in Proc. OFC'09, (San Diego, CA), paper OWG3.

5. P. J. Winzer and R.-J. . Essiambre, “Advanced modulation formats for high-capacity optical transport networks," J. Lightw. Technol. 24, 4711-4728 (2006). 